\title{
WellComp 2021: Fourth International Workshop on Computing for Well-Being
}

\author{
Tadashi Okoshi \\ slash@sfc.keio.ac.jp \\ Keio University \\ Japan
}

\author{
Jin Nakazawa \\ jin@sfc.keio.ac.jp \\ Keio University \\ Japan
}

\author{
JeongGil Ko \\ jeonggil.ko@yonsei.ac.kr \\ Yonsei University \\ Korea
}

\author{
Fahim Kawsar \\ fahim.kawsar@nokia-bell-labs.com \\ Nokia Bell Labs. \\ United Kingdom
}

\author{
Susanna Pirttikangas \\ Susanna.Pirttikangas@oulu.fi \\ University of Oulu \\ Finland
}

\begin{abstract}
With the advancements in ubiquitous computing, ubicomp technology has deeply spread into our daily lives, including office work, home and house-keeping, health management, transportation, or even urban living environments. Furthermore, beyond the initial metrics commonly applied in computing, such as "efficiency" and "productivity", the benefits that people (users) benefit on a wellbeing perspective based on such ubiquitous technology has been greatly emphasized in the recent years. Through the second "WellComp" (Computing for Well-being) workshop, we will intensively discuss about the contribution of ubiquitous computing towards users' well-being that covers both physical, mental, and social wellness (and their combinations), from the viewpoints of various different layers of computing. Having strong international organization members in various ubicomp research domains, WellComp 2021 will bring together researchers and practitioners from the academia and industry to explore versatile topics related to well-being and ubiquitous computing.
\end{abstract}

\section{CCS CONCEPTS}

- Human computer interaction (HCI); • Ubiquitous computing;

\section{KEYWORDS}

Well-being; Physical Wellness, Mental Health, Social Good

\section{ACM Reference Format:}

Tadashi Okoshi, Jin Nakazawa, JeongGil Ko, Fahim Kawsar, and Susanna Pirttikangas. 2021. WellComp 2021: Fourth International Workshop on Computing for Well-Being. In Adjunct Proceedings of the 2021 ACM International Joint Conference on Pervasive and Ubiquitous Computing and Proceedings of the 2021 ACM International Symposium on Wearable Computers (UbiCompISWC '21 Adjunct), September 21-26, 2021, Virtual, USA. ACM, New York, NY, USA, 4 pages. https://doi.org/10.1145/xxxxxxx.Xxxxxxx

Permission to make digital or hard copies of all or part of this work for personal or classroom use is granted without fee provided that copies are not made or distributed for profit or commercial advantage and that copies bear this notice and the full citation on the first page. Copyrights for components of this work owned by others than ACM must be honored. Abstracting with credit is permitted. To copy otherwise, or republish, to post on servers or to redistribute to lists, requires prior specific permission and/or a fee. Request permissions from permissions@acm.org.

UbiComp-ISWC '21 Adjunct, September 21-26, 2021, Virtual, USA

(c) 2021 Association for Computing Machinery.

ACM ISBN xxx-x-xxxx-xxxx-x

https://doi.org/10.1145/xxxxxxx.xxxxxxx

\section{BACKGROUND}

In the advancing ubiquitous computing age, computing technology has already spread into many aspects of our daily lives, such as office work, home and house-keeping, health management, transportation, or even cities. We have been experiencing that much of the influence from those technologies are both contributing to a better quality of life (QoL) of our individual and organizational lives, and causing new types of stress and pain at the same time. In the U.S., life satisfaction has not increased significantly for the last 30 years despite the gross wealth tripling, versatile computing technologies being introduced into their lives [8].

The term "well-being" has recently gained attention as a term that covers our general happiness and even more concrete good conditions in our lives, such as physical, psychological, and social wellness. Even economists and governments are starting to focus on well-being and "Gross National Happiness" as a new metric for measuring the statuses of the nations in addition to the traditional metrics such as GDP and unemployment rate.

An increasing number of researchers, engineers, and people are paying attention to how their work can contribute to better quality of lives, social good, and well-being. This is quite in contrast to the criteria highlighted in the early age of computing, where the focus was on productivity and efficiency. Several trends in the technology field [1-4] are already being observed. Also, the "Positive Computing" community has initiated activities in the human computer interaction (HCI) domain [7], being inspired from positive psychology [9] that focuses on people's psychology under healthy conditions (not in the ill-ed condition). Meanwhile, also in the UbiComp community, two workshops $[5,6]$ have took place, mainly focusing on detecting people's mental illness by using smart and wearable devices.

In spite of such recent activities in academia and the society, unified academic research activities on computing and well-being is anticipated within the ubicomp research community. Active research not only in the HCI domain but in various other ubicomp research areas (systems, mobile/wearable sensing, mobile computing, persuasive applications and services, behavior change, etc.) are needed towards drawing the big picture of "computing for well-being” from different viewpoints and layers of computing. Researchers have been already actively working on human activity recognition (mainly focusing on "physical" activities), psychological state recognition, and even societal activity recognition in 

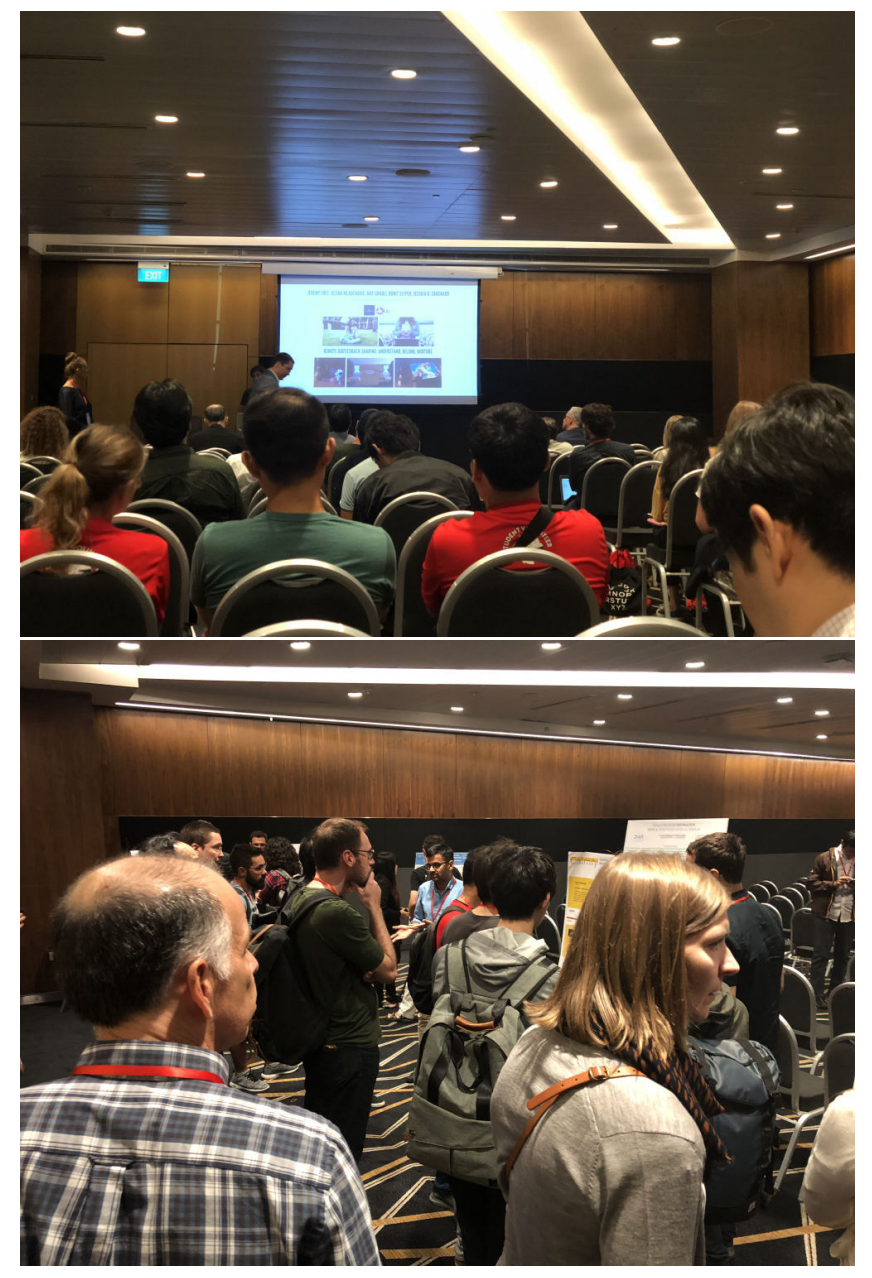

Figure 1: Photos from WellComp 2018 (Oral Presentation Session (upper) and Poster Session (lower)

urban and natural environments. Additional viewpoint of "wellbeing" of people/community in their researches will be possible with new types of applications that comprehensively cover such different types of activity recognition. Ever since Mark Weiser [10] introduced term term of ubiquitous computing, the ubiquity of computing in our daily lives and the society has been certainly progressing. Now it is time for the community to more seriously envision the benefits that such computing technologies can bring.

\section{WELLCOMP 2021 WORKSHOP}

In this workshop, we will bring together people from industry and academia who are active in the areas of activity recognition, mental health, social good, context-awareness and ubiquitous computing. Following three very successful workshops (WellComp 2018, 2019 and 2020) with more than 50 attendees respectively (shown in Figure 1, Figure 2 and Figure 3), the main objective of WellComp 2021 is to share the latest research in various areas in computing, related to users' physical, mental, and social well-being. Expected areas and topics include, but not limited to, systems research, user studies,

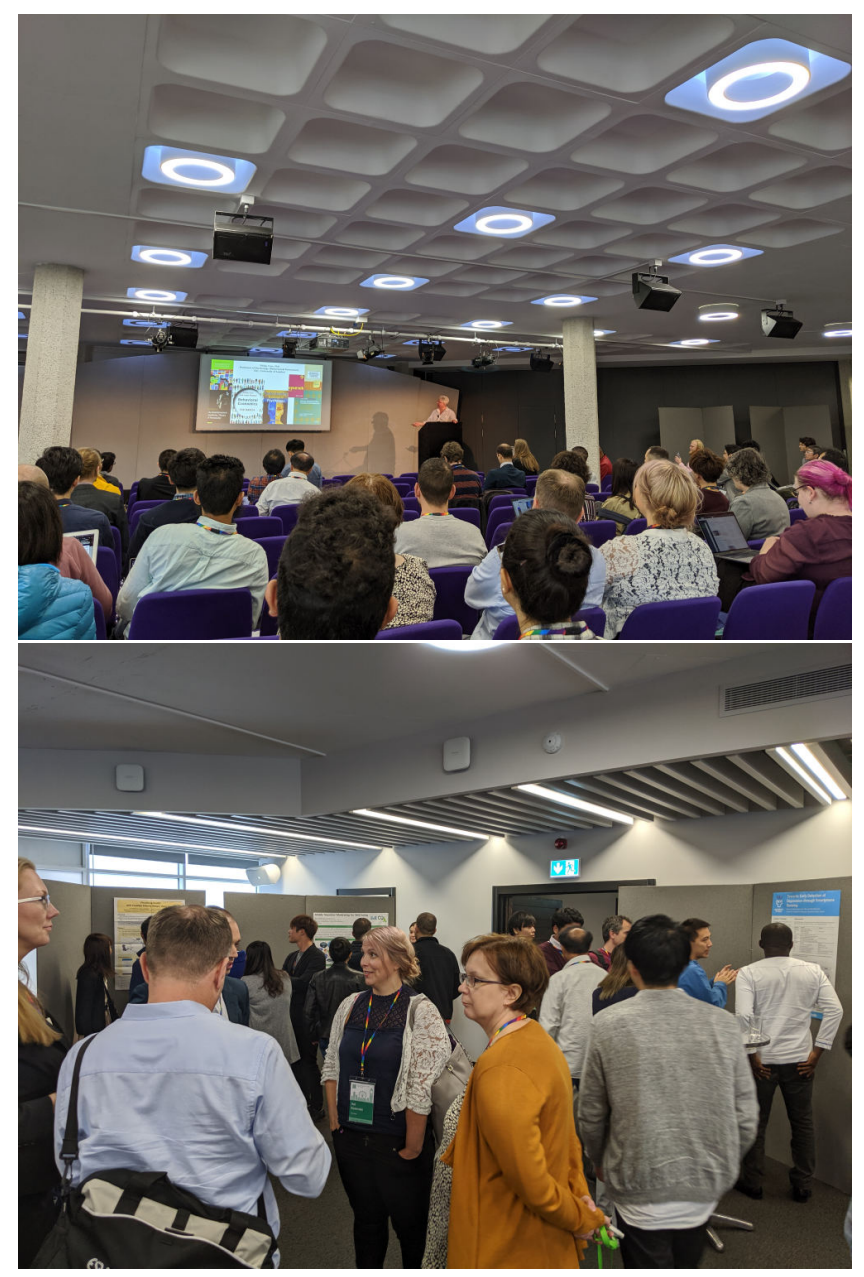

Figure 2: Photos from WellComp 2019 (Keynote presentation by Prof. Philip J. Corr (upper) and Poster Session (lower)

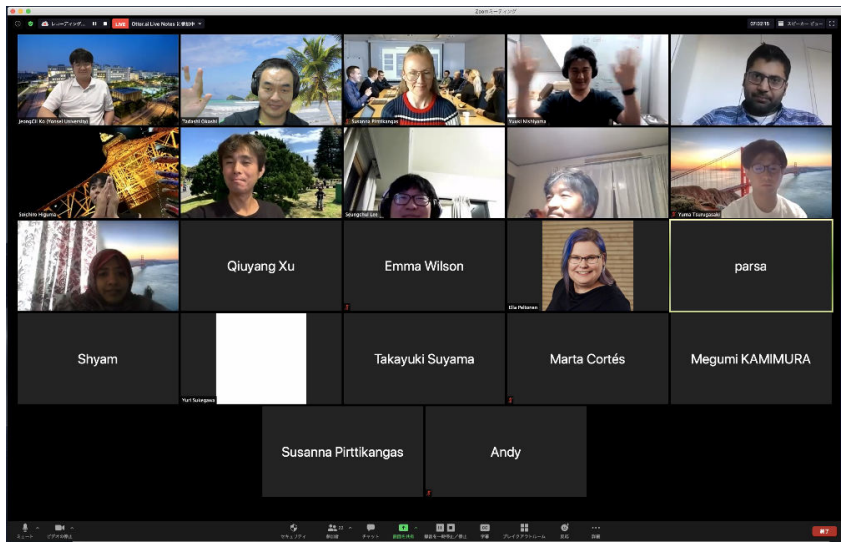

Figure 3: Photos from WellComp 2020 (Virtual Event) 
Table 1: Topics of WellComp Call For Paper

(1) Definition and representation of well-being in computing

(2) Predictive modeling of well-being metric and computational models

(3) Systems with well-being-awareness

(4) Measurement of well-being with ubicomp technologies

(5) Management of physical wellness and well-being

(6) Management of mental health and well-being

(7) Management of social good and well-being

(8) Innovative well-being applications

(9) Behavior design / feedback design for well-being application

(10) Computing and well-being of children, disabilities, or elderly people

(11) People's well-being in various situations (e.g., smart home, smart cities and connected communities, classes)

(12) People's well-being and new types of community driven by ICT (e.g., sharing economy)

networking, social computing, as shown in Table 1. Especially for the fourth workshop, this year's special attention will be paid for "management of personal well-being during COVID-19 pandemic." Relevance to such topics will be considered in the paper review and selection process. Furthermore, the workshop aims to identify future research challenges, research opportunities, and applications of our research outcomes to the society.

Relevance and impact to UbiComp: Emergence of versatile types of computing devices (including IoT), services, and computing venues have been realizing advanced "ubiquity" of computing. WellComp 2021's focus on user's well-being is related to all of the Ubicomp research fields above, and can open new applications to which ubicomp technology can be applied to. Furthermore, the introduction of new applications and insights from the WellComp workshop also has significant potential to impact and contribute to Ubicomp's interest areas horizontally.

Long-term objectives: The workshop will lead to a deeper understanding of well-being and its relationship between ubiquitous computing. The fourth workshop will position the theme within the Ubicomp research community and the definition of "computing for well-being” will continuously be refined. Under such efforts, we plan to build an active and long-lasting community around the workshop's theme. For example, we want to use the workshop's drive to prepare a special issue of a journal, e.g., IEEE Pervasive. We will plan to make an open call for this special issue, but will especially invite workshop participants to submit their work. Furthermore, we consolidate what we find from the workshop (including discussion) into an article which highlights the workshop's main insights.

The workshop's website, along with social media accounts, will be the central venue for us to disseminate such insights from the workshop. We will build our web site under wellcomp. org domain and share all workshop contributions (including papers and discussion results) to the web. Furthermore, social media pages (e.g., Facebook page) will be the bride for us to reach potential authors, participants, and further collaborators.

\section{ORGANIZERS}

WellComp 2021 has a strong international team of researchers in versatile ubicomp-related areas.

- Tadashi Okoshi is associate professor in Faculty of Environment and Information Studies, Keio University. He is actively researching on context and attention-awareness, mobile sensing and interaction, persuasive systems and services for user's higher QoL and behavior changes.

- Jin Nakazawa is an associate professor at Faculty of Environment and Information Studies at Keio University, Japan. His research interest includes middleware systems, distributed systems, ubiquitous computing systems, and life logging.

- JeongGil Ko is an assistant professor in the Department of Software and Computer Engineering at Ajou University. His research interests are in developing embedded and mobile sensing systems with ambient intelligence for IoT and CPS.

- Fahim Kawsar borrows tenets from Social Psychology, learns from Behavioral Economics and applies Computer Science methods to develop human-centered software systems. An experimental computer scientist at heart, he spends most of his time leading the Pervasive Systems research at Nokia Bell Labs, Cambridge. He is intrigued by the power of design and frequently visits TU Delft to teach and debate the influence of data systems in redefining design, and vice versa.

- Susanna Pirttikangas is a senior research fellow and adjunct professor at the Faculty of Information and Electrical Engineering, University of Oulu. In her research, she develops methodology to de-noise, fuse, segment, and classify real-time data streams as well as exploit and test Big Data platforms with a focus on situation awareness as well as business development.

\section{WORKSHOP PAPERS}

Table 1 shows the possible topics of the papers we will ask the workshop authors to contribute. The papers need to be with a length of maximum 4 pages ACM SIGCHI Master Article template. The accepted papers are to be included in the ACM Digital Library. The call for papers will be sent to all relevant mailing lists of several research communities, including those of ubicomp research, HCI, mobile interaction, as well as the IoT, smart city, smart home, and smart vehicle communities. We will distribute our call for papers to various mailing lists of ubicomp-related researcher communities, including ubiquitous, pervasive and mobile computing, HCI, systems research, IoT, distributed systems, or mobile health.

At least two reviewers from our organizers and PC members will peer-review each submission. Our acceptance criteria will be a mixture of relevance, novelty, provocativeness, and research quality. We expect to have a selection of 15-20 accepted papers. Given the theme of this workshop, we are confident to attract a large number of paper submissions which enables us to construct a highquality workshop.

\section{WORKSHOP STRUCTURE}

Following three successful workshops in 2018, 2019 and 2020, we plan WellComp'21 as a single-full-day workshop whose program is shown in Table 2. We're planning to have a keynote lecture 
Table 2: The expected workshop program: mixture of inspiring keynote, paper presentations, and creative group discussions

$\begin{array}{ll}\text { 09:00-09:10 } & \text { Introductions } \\ \text { 09:10-10:00 } & \text { Keynote Talk } \\ \text { 10:00-10:30 } & \text { Coffee Break } \\ \text { 10:30-12:00 } & \text { Presentation } \\ & \text { Session } \\ \text { 12:00-13:00 } & \text { Lunch Break } \\ \text { 13:00-14:30 } & \text { Group Discussion } \\ \text { 14:30-15:00 } & \text { Coffee Break } \\ \text { 15:00-16:00 } & \text { Group Presenta- } \\ & \text { tions and Discus- } \\ & \text { sions } \\ \text { 16:30-17:00 } & \text { Closing Remarks \& } \\ & \text { Future Planning }\end{array}$

by a distinguished researcher focusing in "well-being", followed by a series of informative paper presentations. In the afternoon, we will have an extensive period for intensive discussions. Splitting the participants into multiple groups with specific topics, each group will be asked to come up with answers and solutions to a specific agenda. The initial candidates of the themes will be proposed by the organizers to the participants before the workshop. However, we will finalize the themes according to further inputs from the participants at the venue. The possible themes include (but not limited to) (1) defining "computing for well-being", (2) architecture of a "well-being by design", (3) further significant challenges and collaborative research themes. During the final session, each group will be asked to present their findings, which will then be briefly discussed and summarized. The closing session will summarize the key findings from the workshop, and discuss about future collaborations and activities of the community.

\section{REFERENCES}

[1] 2009. Design for Good. https://www.aiga.org/design-for-good.

[2] 2009. Wisdom 2.0. http://www.wisdom2summit.com/.

[3] 2010. UX for Good. http://www.uxforgood.org/.

[4] 2011. Games for Change. http://www.gamesforchange.org/.

[5] 2016. Mental Health: Sensing and Intervention (2016). https://ubicomp-mentalhealth.github.io/workshop-2016.html.

[6] 2017. Mental Health: Sensing and Intervention (2017). https://ubicomp-mentalhealth.github.io/workshop-2017.html.

[7] Rafael A Calvo and Dorian Peters. 2014. Positive computing: technology for wellbeing and human potential.

[8] John Helliwell, Richard Layard, and Jeffrey Sachs. 2012. World happiness report. The Earth Institute, Columbia University.

[9] Martin EP Seligman and Mihaly Csikszentmihalyi. 2014. Positive psychology: An introduction. In Flow and the foundations of positive psychology. Springer, 279-298.

[10] Mark Weiser. 1996. Ubiquitous Computing. http://www.ubiq.com/hypertext/ weiser/ UbiHome.html. 